\title{
Unique Sequences Containing No $k$-Term Arithmetic Progressions
}

\author{
Tanbir Ahmed \\ Department of Computer Science and Software Engineering \\ Concordia University, Montréal, Canada \\ ta_ahmed@cs. concordia.ca \\ Janusz Dybizbański \\ Institute of Informatics \\ University of Gdańsk, Poland \\ jdybiz@inf.ug.edu.pl \\ Hunter Snevily* \\ Department of Mathematics \\ University of Idaho - Moscow, Idaho, USA \\ snevily@uidaho.edu
}

Submitted: Dec 22, 2012; Accepted: Dec 5, 2013; Published: Dec 17, 2013

Mathematics Subject Classifications: 11B25

\begin{abstract}
In this paper, we are concerned with calculating $r(k, n)$, the length of the longest $k$-Ap free subsequences in $1,2, \ldots, n$. We prove the basic inequality $r(k, n) \leqslant n-$ $\lfloor m / 2\rfloor$, where $n=m(k-1)+r$ and $r<k-1$. We also discuss a generalization of a famous conjecture of Szekeres (as appears in Erdős and Turán [4]) and describe a simple greedy algorithm that appears to give an optimal $k$-AP free sequence infinitely often. We provide many exact values of $r(k, n)$ in the Appendix.
\end{abstract}

\section{Introduction}

Let $\langle n\rangle$ denote the sequence $1,2, \ldots, n$. A subsequence of $\langle n\rangle$ is called $k$-AP free if it does not contain any $k$-term arithmetic progression. Define the following:

$$
\begin{aligned}
r(k, n) & =\text { length of the longest } k \text {-AP free subsequences in }\langle n\rangle, \\
S(k, n) & =\{S \subseteq\{1,2, \ldots, n\}: S \text { is } k \text {-AP free and }|S|=r(k, n)\} \\
b(k, n) & =|S(k, n)|
\end{aligned}
$$

*Hunter Snevily has passed away on November 11, 2013 after his long struggle with Parkinson's disease. We have lost a good friend and colleague. He will be greatly missed and remembered. 
The study of the function $r(3, n)$ was initiated by Erdös and Turán [4]. They determined the values of $r(3, n)$ for $n \leqslant 23$ and $n=41$, proved for $n \geqslant 8$ that

$$
r(3,2 n) \leqslant n
$$

and conjectured that

$$
\lim _{n \rightarrow \infty} r(3, n) / n=0,
$$

which was proved in 1975 by Szemeredi [8]. Erdös and Turán also conjectured that $r(3, n)<n^{1-c}$, which was shown to be false by Salem and Spencer [6], who proved

$$
r(3, n)>n^{1-c / \log \log n}
$$

which was further improved by Behrend [1] to

$$
r(3, n)>n^{1-c / \sqrt{\log n}} .
$$

Recently, Elkin [3] has further improved this lower bound by a factor of $\Theta(\sqrt{\log n})$. The first non-trivial upper bound was due to Roth [5] who proved

$$
r(3, n)<c n / \log \log n
$$

Sharma [7] showed that Erdös and Turán gave the wrong value of $r(3,20)$ and determined the values of $r(3, n)$ for $n \leqslant 27$ and $41 \leqslant n \leqslant 43$. Recently, Dybizbański [2] has computed the exact values of $r(3, n)$ for all $n \leqslant 123$ and proved for $n \geqslant 16$ that

$$
r(3,3 n) \leqslant n .
$$

\subsection{Szekeres' conjecture}

Erdős and Turán [4] noted that there is no 3-term arithmetic progression in the sequence of all numbers $n, 0 \leqslant n \leqslant \frac{1}{2}\left(3^{t}-1\right)$, which do not contain the digit 2 in the ternary scale. Hence for every $t \geqslant 1$,

$$
r\left(3,\left(3^{t}+1\right) / 2\right) \geqslant 2^{t}
$$

as we obtain the 3 -AP-free sequence of length $2^{t}$ in $\left\langle\left(3^{t}+1\right) / 2\right\rangle$ by adding 1 to each of those numbers that does not contain digit 2 in the ternary scale. Szekeres conjectured that for every $t \geqslant 1$,

$$
r\left(3,\left(3^{t}+1\right) / 2\right)=2^{t},
$$

and more generally, for any $t$ and any prime $p$,

$$
r\left(p, \frac{(p-2) p^{t}+1}{p-1}\right)=(p-1)^{t}
$$

A generalization of Szekeres' conjecture will be given in Section 4 . 


\section{A basic inequality for $r(k, n)$}

Let $a p(k, n)$ denote the set of all $k$-APs from the numbers $1,2, \ldots, n$. Let $a p(k, n ; v)$ denote the set of all $k$-APs, each containing $v$, from the numbers $1,2, \ldots, n$. Let $c(k, n)$ and $c(k, n ; v)$ denote $|a p(k, n)|$ and $|a p(k, n ; v)|$, respectively. Let $c_{i}(k, n ; v)$ be the number of $k$-APs, each containing $v$ as the $i$-th element in the AP, from the numbers $1,2, \ldots, n$. Also define $c_{\max }(k, n)$ as the maximum of $c(k, n ; x)$ over $x=1,2, \ldots, n$.

In this section, we determine an exact expression for $c(k, n)$ and an upper-bound for $c_{\text {max }}(k, n)$ to obtain a basic inequality for $r(k, n)$. The following observation is crucial for the proofs in this paper:

\section{Observation 1.}

$$
c_{j}(k, n ; x)= \begin{cases}\lfloor(n-x) /(k-1)\rfloor, & \text { if } j=1 \\ \lfloor(x-1) /(k-1)\rfloor, & \text { if } j=k \\ \min \left\{\left\lfloor\frac{(n-x)}{(k-j)}\right\rfloor,\left\lfloor\frac{(x-1)}{(j-1)}\right\rfloor\right\}, & \text { otherwise. }\end{cases}
$$

and $c(k, n ; x)=\sum_{j=1}^{k} c_{j}(k, n ; x)$.

Example 1. Consider $k=4$ and $n=17$. Here, $r(4,17)=11$ with an example $S=$ $\{1,2,4,5,6,10,12,13,15,16,17\} \in S(4,17)$.

\begin{tabular}{l|rrrrrrrrrrrrrrrrr}
$j / x$ & 1 & 2 & 3 & 4 & 5 & 6 & 7 & 8 & 9 & 10 & 11 & 12 & 13 & 14 & 15 & 16 & 17 \\
\hline 1 & 5 & 5 & 4 & 4 & 4 & 3 & 3 & 3 & 2 & 2 & 2 & 1 & 1 & 1 & 0 & 0 & 0 \\
2 & 0 & 1 & 2 & 3 & 4 & 5 & 5 & 4 & 4 & 3 & 3 & 2 & 2 & 1 & 1 & 0 & 0 \\
3 & 0 & 0 & 1 & 1 & 2 & 2 & 3 & 3 & 4 & 4 & 5 & 5 & 4 & 3 & 2 & 1 & 0 \\
4 & 0 & 0 & 0 & 1 & 1 & 1 & 2 & 2 & 2 & 3 & 3 & 3 & 4 & 4 & 4 & 5 & 5 \\
\hline$c(4,17 ; x)$ & 5 & 6 & 7 & 9 & 11 & 11 & 13 & 12 & 12 & 12 & 13 & 11 & 11 & 9 & 7 & 6 & 5
\end{tabular}

Here $c(4,17 ; x)=c_{1}(4,17 ; x)+c_{2}(4,17 ; x)+c_{3}(4,17 ; x)+c_{4}(4,17 ; x)$. For example, $c(4,17 ; 13)=1+2+4+4$ due to the following 4 -APs in $a p(4,17)$ that contain 13 , namely ap $(4,17 ; 13)$ :

$$
\begin{array}{llll}
\{13,14,15,16\}, & \{12,13,14,15\}, & \{11,13,15,17\}, & \{5,9,13,17\}, \\
\{7,10,13,16\}, & \{9,11,13,15\}, & \{11,12,13,14\}, & \{1,5,9,13\}, \\
\{4,7,10,13\}, & \{7,9,11,13\}, & \{10,11,12,13\} . &
\end{array}
$$

Observation 2. For $x=1,2, \ldots,\lfloor n / 2\rfloor, c(k, n ; x)=c(k, n ; n-x+1)$, that is, the sequence $c(k, n ; x)$ with $x=1,2, \ldots, n$ is symmetric.

Proof. We have the following cases:

(a) For $j=1$, using Observation 1, we have

$$
c_{1}(k, n ; x)=\left\lfloor\frac{n-x}{k-1}\right\rfloor=\left\lfloor\frac{(n-x+1)-1}{k-1}\right\rfloor=c_{k}(k, n ; n-x+1) .
$$


(b) For other values of $j$,

(i) If $\lfloor(n-x) /(k-j)\rfloor \leqslant\lfloor(x-1) /(j-1)\rfloor$, then taking $x^{\prime}=n-x+1$ and $j^{\prime}=k-j+1$, and using Observation 1 .

$$
c_{j}(k, n ; x)=\left\lfloor\frac{(n-x)}{(k-j)}\right\rfloor=\left\lfloor\frac{(n-x+1)-1}{(k-j+1)-1}\right\rfloor=\left\lfloor\frac{\left(x^{\prime}-1\right)}{\left(j^{\prime}-1\right)}\right\rfloor=c_{j^{\prime}}\left(k, n ; x^{\prime}\right) .
$$

The last equality follows from the fact that

$$
\left\lfloor\frac{\left(x^{\prime}-1\right)}{\left(j^{\prime}-1\right)}\right\rfloor \leqslant\left\lfloor\frac{(x-1)}{(j-1)}\right\rfloor=\left\lfloor\frac{\left(n-x^{\prime}\right)}{\left(k-j^{\prime}\right)}\right\rfloor .
$$

(ii) If $\lfloor(n-x) /(k-j)\rfloor>\lfloor(x-1) /(j-1)\rfloor$, then (similar as $(i))$

$$
c_{j}(k, n ; x)=c_{k-j+1}(k, n ; n-x+1) .
$$

Therefore, $c(k, n ; x)$ with $x=1,2, \ldots, n$ is symmetric.

Lemma 1. Given positive integers $k$ and $n$, let $2 \leqslant j \leqslant k-1, n=m(k-1)+r$ with $0 \leqslant r \leqslant k-2$. Then $c_{j}(k, n ; x)$ equals

(a) $\lfloor(x-1) /(j-1)\rfloor$ if $x=1,2, \ldots, m(j-1)$,

(b) $\lfloor(n-x) /(k-j)\rfloor$ if $x=n-m(k-j)+1, n-m(k-j)+2, \ldots, n$,

(c) $m$ otherwise, that is, for $x=m(j-1)+1, \ldots, n-m(k-j)$.

Proof. (a) Take $y \leqslant m(j-1)$ and assume

$$
c_{j}(k, n ; y)=\lfloor(n-y) /(k-j)\rfloor,
$$

that is, $\lfloor(n-y) /(k-j)\rfloor<\lfloor(y-1) /(j-1)\rfloor$.

Since $y \leqslant m(j-1)$, we have $(y-1) \leqslant m(j-1)-1$, and hence

$$
\left\lfloor\frac{y-1}{j-1}\right\rfloor \leqslant m-1 \text {. }
$$

Again, since $y \leqslant m(j-1)$, we have

$$
n-y \geqslant n-m(j-1)=m(k-j)+r,
$$

which implies

$$
\left\lfloor\frac{n-y}{k-j}\right\rfloor \geqslant m
$$

Now, we have the following contradiction

$$
m \leqslant\left\lfloor\frac{n-y}{k-j}\right\rfloor<\left\lfloor\frac{y-1}{j-1}\right\rfloor \leqslant m-1 .
$$


(b) Take $y \geqslant n-m(k-j)+1$ and assume

$$
c_{j}(k, n ; y)=\lfloor(y-1) /(j-1)\rfloor,
$$

that is, $\lfloor(n-y) /(k-j)\rfloor>\lfloor(y-1) /(j-1)\rfloor$. Similar reasoning as (a) leads to a contradiction.

(c) Here, $n-m(k-j)$ can be written as $m(j-1)+r$ and $m(j-1)+1$ can be written as $n-m(k-j)-(r-1)$. There are exactly $r$ elements in $m(j-1)+1, \ldots, n-m(k-j)$, and for any $x$ in this range,

$$
c_{j}(k, n ; x)=\lfloor(x-1) /(j-1)\rfloor=\lfloor(n-x) /(k-j)\rfloor=m .
$$

Lemma 2. Given positive integers $k$ and $n$, let $n=m(k-1)+r$ with $0 \leqslant r \leqslant k-2$. Denote a sequence $a, a, \ldots, a$ with $a$ repeated $b$ times as $a^{b}$, and consider $a^{0}$ to be an empty sequence. Then for $1 \leqslant j \leqslant k$, the sequence $c_{j}(k, n ; x)$ with $1 \leqslant x \leqslant n$ has the form

$$
0^{j-1} 1^{j-1} \cdots(m-1)^{j-1} m^{r}(m-1)^{k-j}(m-2)^{k-j} \cdots 0^{k-j} .
$$

Proof. Using Observation 1, we have for $j=1$ :

$$
c_{1}(k, n ; x)=\left\lfloor\frac{n-x}{k-1}\right\rfloor=\left\lfloor m+\frac{r-x}{k-1}\right\rfloor=m+\left\lfloor\frac{r-x}{k-1}\right\rfloor,
$$

and more specifically,

$$
c_{1}(k, n ; x)= \begin{cases}m, & \text { for } x=1,2, \ldots, r \\ (m-1), & \text { for } x=r+1, r+2, \ldots, r+(k-1) \\ (m-2), & \text { for } x=r+(k-1)+1, r+(k-1)+2, \ldots, r+2(k-1) \\ & \vdots, \\ 1, & \text { for } x=r+(m-2)(k-1)+1, \ldots, n-(k-1) \\ 0, & \text { for } x=r+(m-1)(k-1)+1, \ldots, n .\end{cases}
$$

Hence, the sequence $c_{1}(k, n ; x)$ with $x=1,2, \ldots, n$ is

$$
m^{r}(m-1)^{k-1}(m-2)^{k-1} \cdots 1^{k-1} 0^{k-1} .
$$

Similarly, for $j=k$, we have

$$
c_{k}(k, n ; x)=\left\lfloor\frac{x-1}{k-1}\right\rfloor
$$

and more specifically,

$$
c_{k}(k, n ; x)= \begin{cases}0, & \text { for } x=1,2, \ldots, k-1 \\ 1, & \text { for } x=(k-1)+1,(k-1)+2, \ldots, 2(k-1) ; \\ 2, & \text { for } x=2(k-1)+1,2(k-1)+2, \ldots, 3(k-1) ; \\ & \vdots, \\ (m-2), & \text { for } x=(m-2)(k-1)+1, \ldots,(m-1)(k-1) ; \\ (m-1), & \text { for } x=(m-1)(k-1)+1, \ldots, m(k-1) ; \\ m, & \text { for } x=m(k-1)+1, \ldots, n .\end{cases}
$$


and hence the sequence $c_{k}(k, n ; x)$ with $x=1,2, \ldots, n$ is

$$
0^{k-1} 1^{k-1} \cdots(m-2)^{k-1}(m-1)^{k-1} m^{r} .
$$

For $2 \leqslant j \leqslant k-1$, by Lemma 1, we have

\begin{tabular}{c|c}
\hline$x$ & $c_{j}(k, n ; x)$ \\
\hline $1,2, \ldots, j-1$ & $\lfloor(x-1) /(j-1)\rfloor=0$ \\
$(j-1)+1,(j-1)+2, \ldots, 2(j-1)$ & $\lfloor(x-1) /(j-1)\rfloor=1$ \\
$\vdots$ & $\vdots$ \\
$(m-2)(j-1)+1,(m-2)(j-1)+2, \ldots,(m-1)(j-1)$ & $\lfloor(x-1) /(j-1)\rfloor=m-2$ \\
$(m-1)(j-1)+1,(m-1)(j-1)+2, \ldots, m(j-1)$ & $\lfloor(x-1) /(j-1)\rfloor=m-1$ \\
\hline$m(j-1)+1, m(j-1)+2, \ldots, n-m(k-j)$ & $m$ \\
\hline$n-m(k-j)+1, \ldots, n-(m-1)(k-j)$ & $\lfloor(n-x) /(k-j)\rfloor=m-1$ \\
$n-(m-1)(k-j)+1, \ldots, n-(m-2)(k-j)$ & $\lfloor(n-x) /(k-j)\rfloor=m-2$ \\
$\vdots$ & $\vdots$ \\
$n-2(k-j)+1, n-2(k-j)+2, \ldots, n-(k-j)$ & $\lfloor(n-x) /(k-j)\rfloor=1$ \\
$n-(k-j)+1, n-(k-j)+2, \ldots, n$ & $\lfloor(n-x) /(k-j)\rfloor=0$ \\
\hline
\end{tabular}

Hence, we get the sequence $c_{j}(k, n ; x)$ for $x=1,2, \ldots, n$ as

$$
0^{j-1} 1^{j-1} \cdots(m-1)^{j-1} m^{r}(m-1)^{k-j}(m-2)^{k-j} \cdots 0^{k-j} .
$$

Corollary 1. Given positive integers $k$ and $n$, let $m=\lfloor n /(k-1)\rfloor$ and $n=m(k-1)+r$. Then

$$
c(k, n)=\left(\begin{array}{c}
m \\
2
\end{array}\right)(k-1)+m r .
$$

Proof. From the definition of $c_{1}(k, n ; x)$ in Lemma 2 , for $1 \leqslant x \leqslant n$, we have,

$$
\begin{aligned}
c(k, n) & =\sum_{x=1}^{n-k+1} c_{1}(k, n ; x) \\
& =\sum_{x=1}^{r} c_{1}(k, n ; x)+\left[\sum_{x=r+1}^{r+(k-1)} c_{1}(k, n ; x)+\right. \\
& \sum_{x=r+(k-1)+1}^{r+2(k-1)} c_{1}(k, n ; x)+\cdots+\sum_{x=r+(m-2)(k-1)+1}^{n-k+1=n-(k-1)=r+(m-1)(k-1)} c_{1}(k, n ; x) \\
= & m r+[(m-1)+(m-2)+\cdots+2+1](k-1) \\
& =\left(\begin{array}{c}
m \\
2
\end{array}\right)(k-1)+m r .
\end{aligned}
$$


Corollary 2. Given positive integers $k$ and $n$, let $n=m(k-1)+r$ with $r<k-1$ and $1 \leqslant j \leqslant k$. Then for $x=1,2, \ldots, n$,

$$
c_{j}(k, n ; x) \leqslant m .
$$

Proof. Follows from Lemmas 1 and 2 .

Trivially, $c_{\max }(k, n) \leqslant m k$. The following Corollary slightly improves the bound.

Corollary 3. Given positive integers $k$ and $n$, let $n=m(k-1)+r$ with $r<k-1$ and $1 \leqslant j \leqslant k$. Then

$$
c_{\text {max }}(k, n) \leqslant m(k-1)
$$

Proof. For any $x \in\{1,2, \ldots, n\}$, using the definitions of $c_{1}(k, n ; x)$ and $c_{k}(k, n ; x)$ from Lemma 2, we have,

\begin{tabular}{c|c}
\hline$x$ & $c_{1}(k, n ; x)+c_{k}(k, n ; x)$ \\
\hline $1,2, \ldots, r$ & $m+0=m$ \\
$r+0(k-1)+1, r+0(k-1)+2, \ldots, k-1$ & $(m-1)+0=m-1$ \\
$1+(k-1), 2+(k-1), \ldots, r+1(k-1)$ & $(m-1)+1=m$ \\
$r+1(k-1)+1, r+1(k-1)+2, \ldots, 2(k-1)$ & $(m-2)+1=m-1$ \\
$1+2(k-1), 2+2(k-1), \ldots, r+2(k-1)$ & $\vdots$ \\
$\vdots$ & $(m-2)+2=m$ \\
$r+(m-2)(k-1)+1, r+(m-2)(k-1)+2, \ldots,(m-1)(k-1)$ & $1+(m-2)=m-1$ \\
$1+(m-1)(k-1), 2+(m-1)(k-1), \ldots, r+(m-1)(k-1)$ & $1+(m-1)=m$ \\
$r+(m-1)(k-1)+1, r+(m-1)(k-1)+2, \ldots, m(k-1)$ & $0+(m-1)=m-1$ \\
$1+m(k-1), 2+m(k-1), \ldots, r+m(k-1)$, & $0+m=m$ \\
\hline
\end{tabular}

Hence, using $c_{1}(k, n ; x)+c_{k}(k, n ; x) \leqslant m$ and $c_{j}(k, n ; x) \leqslant m$ (by Corollary 2) for $1 \leqslant j \leqslant k$, we have

$$
\begin{aligned}
c(k, n ; x) & =c_{1}(k, n ; x)+c_{k}(k, n ; x)+\sum_{j=2}^{k-1} c_{j}(k, n ; x) \\
& \leqslant m+m(k-2)=m(k-1) .
\end{aligned}
$$

Therefore, $c_{\max }(k, n) \leqslant m(k-1)$.

It can be observed that the upper bound in Corollary 3 is the best possible for $c_{\text {max }}(k, n)$. The following theorem gives an upper bound of $r(k, n)$, which is very close to actual values (see Appendix C for experimental results).

Theorem 1. Given positive integers $k$ and $n$, let $m=\lfloor n /(k-1)\rfloor$ and $n=m(k-1)+r$ where $r<k-1$. Then

$$
r(k, n) \leqslant n-\lfloor m / 2\rfloor .
$$


Proof. Using Corollaries 2 and 3 , we have

$$
\begin{aligned}
r(k, n) & \leqslant n-\left\lceil\frac{c(k, n)}{c_{\max }(k, n)}\right\rceil \\
& \leqslant n-\left\lceil\frac{m(m-1)(k-1) / 2+m r}{m(k-1)}\right\rceil \\
& =n-\left\lceil\frac{m-1}{2}+\frac{r}{k-1}\right\rceil=n-f(m, k, r),(\text { say })
\end{aligned}
$$

It can be observed that

$$
f(m, k, r)= \begin{cases}y+1, & \text { if } m=2 y+1 \\ y, & \text { if } m=2 y \text { and } 2 r \leqslant k-1 \\ y+1, & \text { if } m=2 y \text { and } 2 r>k-1\end{cases}
$$

Hence,

$$
r(k, n) \leqslant n-\lfloor m / 2\rfloor .
$$

Conjecture 1. For every positive integer $k \geqslant 3$ and positive integer $n, c_{\max }(k, n)$ is eventually periodic.

For example, for $k=5$, the length of the period is 24 and the periodic increase in the value of $c_{\max }(k, n)$ is 20 , as indicated in the following table:

\begin{tabular}{c|c||c|c||c|c||c|c||c|c||c}
\hline$n$ & $c_{\max }(5, n)$ & $n$ & $c_{\max }(5, n)$ & $n$ & $c_{\max }(5, n)$ & $n$ & $c_{\max }(5, n)$ & $n$ & $c_{\max }(5, n)$ & \\
\hline 25 & 20 & 49 & 40 & 73 & 60 & 97 & 80 & 121 & 100 & $\cdots$ \\
26 & 20 & 50 & 40 & 74 & 60 & 98 & 80 & 122 & 100 & $\ldots$ \\
27 & 20 & 51 & 40 & 75 & 60 & 99 & 80 & 123 & 100 & $\ldots$ \\
28 & 21 & 52 & 41 & 76 & 61 & 100 & 81 & 124 & 101 & $\ldots$ \\
29 & 22 & 53 & 42 & 77 & 62 & 101 & 82 & 125 & 102 & $\ldots$ \\
30 & 22 & 54 & 42 & 78 & 62 & 102 & 82 & 126 & 102 & $\ldots$ \\
$\vdots$ & $\vdots$ & $\vdots$ & $\vdots$ & $\vdots$ & $\vdots$ & $\vdots$ & $\vdots$ & $\vdots$ & $\vdots$ & $\vdots$ \\
47 & 37 & 71 & 57 & 95 & 77 & 119 & 97 & 143 & 117 & $\ldots$ \\
48 & 37 & 72 & 57 & 96 & 77 & 120 & 97 & 144 & 117 & $\ldots$ \\
\hline
\end{tabular}

Conjecture 2. Given an odd positive integer $k$, the size of the largest subset $U$ of $\{1,2, \ldots, n\}$ for any positive integer $n$, with each $x \in U$ having the same $c(k, n ; x)$, is bounded from above by a constant $f(k) \leqslant k^{2}$.

The implications of Conjecture 2 being true is as follows:

Let $w=c_{\max }(k, n)$ and consider the largest $\ell$ such that

$$
g(k, \ell, w)=f(k)(w+(w-1)+\cdots+(w-(\ell-1)))
$$

has the property $c(k, n)-g(k, \ell, w) \geqslant f(k) \ell(\ell-1) / 2$. Then 


$$
\begin{aligned}
r(k, n) & \leqslant n-\left\lceil\frac{c(k, n)}{w}\right\rceil \leqslant n-\left\lceil\frac{g(k, \ell, w)+f(k) \ell(\ell-1) / 2}{w}\right\rceil \\
& =n-f(k) \ell .
\end{aligned}
$$

See Appendix A for data supporting Conjectures 1 and 2 .

\section{Unimodality lemmas}

A sequence is called unimodal if it is first increasing and then decreasing. In this section, we prove some lemmas on sequences regarding $c(k, n ; x)$ for $k \geqslant 3$.

Lemma 3. Given positive integers $k$ and $n$, for any $j \in\{2,3, \ldots, k-1\}$, the sequence $c_{j}(k, n ; x)$ with $x=1,2, \ldots, n$ is unimodal.

Proof. Follows directly from Lemma 2.

Lemma 4. The sequence $c(3, n ; i)$ with $i=1,2, \ldots, n$ is unimodal.

Proof. From Observation 1 .

$$
c_{j}(3, n ; x)= \begin{cases}\lfloor(n-x) / 2\rfloor, & \text { if } j=1 ; \\ x-1, & \text { if } j=2 \text { and } x \leqslant\lfloor n / 2\rfloor ; \\ n-x, & \text { if } j=2 \text { and } x>\lfloor n / 2\rfloor ; \\ \lfloor(x-1) / 2\rfloor, & \text { if } j=3 .\end{cases}
$$

By Observation 2, $c(3, n ; i)$ equals $c(3, n ; n-i+1)$ for $i=1,2, \ldots,\lfloor n / 2\rfloor$.

Now, we consider the following two cases:

1. $(n=2 m)$. For $i=1,2, \ldots, m-1$, we have

$$
c_{2}(3, n ; i+1)=c_{2}(3, n ; i)+1,
$$

and also for $i=1,2, \ldots, m$,

$$
c_{1}(3, n ; i)+c_{3}(3, n ; i)=\left\lfloor\frac{2 m-i}{2}\right\rfloor+\left\lfloor\frac{i-1}{2}\right\rfloor=\left\lfloor m-\frac{i}{2}\right\rfloor+\left\lfloor\frac{i}{2}-\frac{1}{2}\right\rfloor .
$$

If $i=2 j(j \geqslant 1)$, then

$$
c_{1}(3, n ; i)+c_{3}(3, n ; i)=(m-j)+\left\lfloor j-\frac{1}{2}\right\rfloor=(m-j)+(j-1)=m-1 .
$$

If $i=2 j+1(j \geqslant 0)$, then

$$
\begin{aligned}
c_{1}(3, n ; i)+c_{3}(3, n ; i) & =\left\lfloor m-j-\frac{1}{2}\right\rfloor+\left\lfloor\frac{(2 j+1)-1}{2}\right\rfloor \\
& =(m-j-1)+j=m-1 .
\end{aligned}
$$

Therefore, for $i=1,2, \ldots, m-1$,

$$
c(3, n ; i+1)=(m-1)+c_{2}(3, n ; i)+1=c(3, n ; i)+1 .
$$


2. $(n=2 m+1)$. For $i=1,2, \ldots, m$, we have

$$
c_{2}(3, n ; i+1)=c_{2}(3, n ; i)+1
$$

and also

$$
c_{1}(3, n ; i)+c_{3}(3, n ; i)=\left\lfloor\frac{2 m+1-i}{2}\right\rfloor+\left\lfloor\frac{i-1}{2}\right\rfloor=\left\lfloor m+\frac{1}{2}-\frac{i}{2}\right\rfloor+\left\lfloor\frac{i}{2}-\frac{1}{2}\right\rfloor .
$$

If $i=2 j(j \geqslant 1)$, then

$$
c_{1}(3, n ; i)+c_{3}(3, n ; i)=\left\lfloor m+\frac{1}{2}-j\right\rfloor+\left\lfloor j-\frac{1}{2}\right\rfloor=(m-j)+(j-1)=m-1 .
$$

If $i=2 j+1(j \geqslant 0)$, then

$$
\begin{aligned}
c_{1}(3, n ; i)+c_{3}(3, n ; i) & =\left\lfloor m+\frac{1}{2}-j-\frac{1}{2}\right\rfloor+\left\lfloor\frac{(2 j+1)-1}{2}\right\rfloor \\
& =(m-j)+j=m .
\end{aligned}
$$

Therefore, for $i=1,2, \ldots, m$,

- If $i$ is odd, then

$$
\begin{aligned}
c(3, n ; i+1) & =c_{1}(3, n ; i+1)+c_{2}(3, n ; i+1)+c_{3}(3, n ; i+1) \\
& =c_{2}(3, n ; i)+1+(m-1)=c_{2}(3, n ; i)+m \\
& =c_{2}(3, n ; i)+c_{1}(3, n ; i)+c_{3}(3, n ; i)=c(3, n ; i) .
\end{aligned}
$$

- If $i$ is even, then

$$
\begin{aligned}
c(3, n ; i+1) & =c_{1}(3, n ; i+1)+c_{2}(3, n ; i+1)+c_{3}(3, n ; i+1) \\
& =c_{2}(3, n ; i)+1+m=c_{2}(3, n ; i)+(m-1)+2 \\
& =c_{2}(3, n ; i)+c_{1}(3, n ; i)+c_{3}(3, n ; i)+2=c(3, n ; i)+2 .
\end{aligned}
$$

Hence, $c(3, n ; i)$ with $i=1,2, \ldots, n$ is unimodal.

Lemma 5. For $k \geqslant 4$, there are infinitely many $n$ such that the sequence $c(k, n ; i)$ with $i=1,2, \ldots, n$ is unimodal.

Proof. We show that $c(k, n ; i)$ for $1 \leqslant i \leqslant n$ with $n=\operatorname{lcm}\{1,2, \ldots, k-1\} \cdot m$ (where $m \geqslant 1)$ is unimodal. Since $n$ is even, $n / 2$ is an integer. By Observation 2 , the sequence $c(k, n ; i)$ with $1 \leqslant i \leqslant n$ is symmetric. So assume $i \leqslant n / 2$. Let $l c m\{1,2, \ldots, k-1\}$ be equal to $h_{r} \cdot r$ with $2 \leqslant r \leqslant k-1$, and $i \equiv s(\bmod k-1)$ with $t=\lfloor i /(k-1)\rfloor$. Now,

$$
\begin{aligned}
c_{1}(k, n ; i) & =\left\lfloor\frac{(n-i)}{(k-1)}\right\rfloor=\left\lfloor m h_{k-1}-\frac{i}{(k-1)}\right\rfloor=\left\lfloor m h_{k-1}-t-\frac{s}{k-1}\right\rfloor \\
c_{1}(k, n ; i+1) & =\left\lfloor\frac{(n-i-1)}{(k-1)}\right\rfloor=\left\lfloor m h_{k-1}-\frac{i+1}{(k-1)}\right\rfloor=\left\lfloor m h_{k-1}-t-\frac{s+1}{k-1}\right\rfloor
\end{aligned}
$$


Therefore,

$$
c_{1}(k, n ; i+1)= \begin{cases}c_{1}(k, n ; i)-1, & \text { if } s=0 \\ c_{1}(k, n ; i), & \text { otherwise }\end{cases}
$$

Similarly,

$$
c_{k}(k, n ; i+1)= \begin{cases}c_{k}(k, n ; i)+1, & \text { if } s=0 ; \\ c_{k}(k, n ; i), & \text { otherwise. }\end{cases}
$$

Hence, $c_{1}(k, n ; i)+c_{k}(k, n ; i)$ remains constant for $1 \leqslant i \leqslant n$.

Again, for $1 \leqslant i \leqslant n / 2$, we have

$$
i-1 \leqslant n-i .
$$

For $2 \leqslant j \leqslant k-1$, we want to show

$$
c_{j}(k, n ; i+1)+c_{k-j+1}(k, n ; i+1) \geqslant c_{j}(k, n ; i)+c_{k-j+1}(k, n ; i) .
$$

Assume $j>\lfloor k / 2\rfloor$. This implies $k-j \leqslant j-1$. Since $i-1 \leqslant n-i$, we have

$$
\left\lfloor\frac{(i-1)}{(j-1)}\right\rfloor \leqslant\left\lfloor\frac{(n-i)}{(k-j)}\right\rfloor .
$$

So for $1 \leqslant i \leqslant n / 2-1$, and considering $i \equiv s(\bmod j-1)$ and $t=\lfloor i /(j-1)\rfloor$, we have,

$$
\begin{aligned}
c_{j}(k, n ; i+1) & =\left\lfloor\frac{i}{j-1}\right\rfloor=t \\
c_{j}(k, n ; i) & =\left\lfloor\frac{i-1}{j-1}\right\rfloor=\left\lfloor t+\frac{s-1}{j-1}\right\rfloor= \begin{cases}t-1, & \text { if } s=0 ; \\
t, & \text { if } s \geqslant 1 .\end{cases}
\end{aligned}
$$

Take $j^{\prime}=k-j+1$, and then $j^{\prime}-1 \leqslant k-j^{\prime}$. If $\left\lfloor(i-1) /\left(j^{\prime}-1\right)\right\rfloor \leqslant\left\lfloor(n-i) /\left(k-j^{\prime}\right)\right\rfloor$, then

$$
0 \leqslant c_{j^{\prime}}(k, n ; i+1)-c_{j^{\prime}}(k, n ; i) \leqslant 1
$$

else

$$
\begin{aligned}
c_{j^{\prime}}(k, n ; i) & =\left\lfloor\frac{n-i}{k-j^{\prime}}\right\rfloor=\left\lfloor\frac{n-i}{j-1}\right\rfloor=\left\lfloor m h_{j-1}-t-\frac{s}{j-1}\right\rfloor \\
& = \begin{cases}\left(m h_{j-1}-t\right), & \text { if } s=0 ; \\
\left(m h_{j-1}-t-1\right), & \text { otherwise. }\end{cases} \\
c_{j^{\prime}}(k, n ; i+1) & =\left\lfloor\frac{n-i-1}{k-j^{\prime}}\right\rfloor=\left\lfloor m h_{j-1}-t-\frac{s+1}{j-1}\right\rfloor=\left(m h_{j-1}-t-1\right)
\end{aligned}
$$

Therefore,

$$
c_{j}(k, n ; i+1)+c_{j^{\prime}}(k, n ; i+1) \geqslant c_{j}(k, n ; i)+c_{j^{\prime}}(k, n ; i) .
$$

So the sequence $c(k, n ; x)$ with $1 \leqslant x \leqslant n / 2$ is non-decreasing and hence the sequence $c(k, n ; x)$ with $1 \leqslant x \leqslant n$ is unimodal for infinitely many $n$. 


\section{Uniqueness conjectures}

In this section, we generalize Szekeres' conjecture and provide a construction for the lower bound. We also provide a construction algorithm for $r(k, n)$. Define

$$
J(k, L)=\{(n, m): n \leqslant L, r(k, n)=m \text { and } b(k, n)=1\} .
$$

We have the following experimental data, based on which we formulate Conjectures 3 and 4 .

$$
\begin{aligned}
J(3,123)= & \{(2,2),(5,4),(14,8),(30,12),(41,16),(74,22),(84,24),(104,28), \\
& (114,30),(122,32)\} \\
J(5,105)= & (2,2),(3,3),(4,4),(9,8),(14,12),(19,16),(44,32),(69,48),(94,64)\}, \\
J(7,139)= & (2,2),(3,3),(4,4),(5,5),(6,6),(13,12),(20,18),(27,24),(34,30), \\
& (41,36),(90,72),(139,108)\} \\
J(11,117)= & (2,2),(3,3),(4,4),(5,5),(6,6),(7,7),(8,8),(9,9),(10,10),(21,20), \\
& (32,30),(43,40),(54,50),(65,60),(76,70),(87,80),(98,90),(109,100)\}, \\
J(13,161)= & \{(2,2),(3,3),(4,4),(5,5),(6,6),(7,7),(8,8),(9,9),(10,10),(11,11), \\
& (12,12),(25,24),(38,36),(51,48),(64,60),(77,72),(90,84),(103,96), \\
& (116,108),(129,120),(142,132),(155,144)\} .
\end{aligned}
$$

Conjecture 3 (The Uniqueness Conjecture). Consider a prime $p \geqslant 3$ and an integer $t \geqslant 1$. Then for $1 \leqslant i \leqslant p-1$,

$$
r\left(p, \frac{(i p-i-1) p^{t}+1}{(p-1)}\right)=i \cdot(p-1)^{t},
$$

and $b(p, x)=1$ where $1 \leqslant x \leqslant p-2$ or else

$$
x=\frac{(i p-i-1) p^{t}+1}{(p-1)} .
$$

It can be observed that Szekeres' conjecture is a special case of Conjecture 3 with $i=1$.

Conjecture 4 (Strong Uniqueness Conjecture). Consider a prime $p>3$ and an integer $t \geqslant 1$. Then $b(p, x)=1$ if and only if $1 \leqslant x \leqslant p-2$ or else

$$
x=\frac{(i p-i-1) p^{t}+1}{(p-1)}
$$

with $1 \leqslant i \leqslant p-1$.

See Appendix C for data supporting Conjectures 3 and 4 . 


\subsection{Construction for the lower-bound of Conjecture 3}

For a prime $p>3$ and $1 \leqslant i \leqslant p-1$, take

$$
n=\frac{(i p-i-1) p^{t}+1}{p-1}=i p^{t}-p^{t-1}-p^{t-2}-\cdots-p-1 .
$$

We can construct a $p$-AP free subset of $\{1,2, \ldots, n\}$ of size $i \cdot(p-1)^{t}$ as follows:

$$
\begin{aligned}
T_{0} & =\{1,2, \ldots, n\}, \\
T_{1} & =T_{0}-\{j: j \equiv 0 \quad(\bmod p)\}=T_{0}-S_{0}, \\
T_{2} & =T_{1}-\left\{j_{1} p^{2}-p+j_{2}: 1 \leqslant j_{1} \leqslant\left\lfloor n / p^{2}\right\rfloor, 1 \leqslant j_{2} \leqslant p-1\right\}=T_{1}-S_{1}, \\
T_{3} & =T_{2}-\left\{j_{1} p^{3}-p^{2}+j_{2} p-j_{3}: 1 \leqslant j_{1} \leqslant\left\lfloor n / p^{3}\right\rfloor, 1 \leqslant j_{2}, j_{3} \leqslant p-1\right\}=T_{2}-S_{2}, \\
T_{4} & =T_{3}-\left\{j_{1} p^{4}-p^{3}+j_{2} p^{2}-j_{3} p+j_{4}: 1 \leqslant j_{1} \leqslant\left\lfloor n / p^{4}\right\rfloor, 1 \leqslant j_{2}, j_{3}, j_{4} \leqslant p-1\right\} \\
& =T_{3}-S_{3}, \\
& \vdots \\
T_{t} & =T_{t-1}-\left\{j_{1} p^{t}-p^{t-1}+\sum_{\ell=2}^{t} p^{t-\ell} j_{\ell}(-1)^{\ell}: 1 \leqslant j_{1} \leqslant\left\lfloor n / p^{t}\right\rfloor, 1 \leqslant j_{2}, j_{3}, \ldots, j_{t} \leqslant p-1\right\} \\
& =T_{t-1}-S_{t-1} .
\end{aligned}
$$

It can be observed that

$$
\begin{aligned}
\left|S_{0}\right| & =\lfloor n / p\rfloor=i p^{t-1}-p^{t-2}-\cdots-p-2, \\
\left|S_{1}\right| & =(p-1)\left\lfloor n / p^{2}\right\rfloor=(p-1)\left(i p^{t-2}-p^{t-3}-\cdots-p-2\right), \\
\left|S_{2}\right| & =(p-1)^{2}\left\lfloor n / p^{3}\right\rfloor=(p-1)^{2}\left(i p^{t-3}-p^{t-4}-\cdots-p-2\right), \\
& \vdots \\
\left|S_{t-1}\right| & =(p-1)^{t-1}\left\lfloor n / p^{t}\right\rfloor=(p-1)^{t-1}(i-1) .
\end{aligned}
$$

Lemma 6. The $S_{\ell}$ 's for $0 \leqslant \ell \leqslant t-1$ are disjoint.

Proof. Each element in $S_{0}$ is divisible by $p$, and no element in any other $S_{\ell}$ is divisible by $p$. So $S_{0}$ is disjoint from every other $S_{\ell}$. For $2 \leqslant \ell \leqslant t-1$,

$$
S_{\ell}=\left\{\left(x p+(-1)^{\ell-1} j_{\ell+1}\right) \in T_{0}: x \in S_{\ell-1}, 1 \leqslant j_{\ell+1} \leqslant p-1\right\},
$$

and hence $S_{\ell} \cap S_{u}=\emptyset$ for $1 \leqslant u \leqslant \ell-1$.

Lemma 7. For a prime $p>3$ and $1 \leqslant i \leqslant p-1$,

$$
\left|T_{t}\right|=i \cdot(p-1)^{t} .
$$


Proof. We can write the summation $\sum_{j=0}^{t-1}\left|S_{j}\right|$ as follows:

$$
\begin{aligned}
\sum_{j=0}^{t-1}\left|S_{j}\right| & =i p^{t-1}\left(\sum_{a=0}^{t-1}\left(\begin{array}{l}
a \\
0
\end{array}\right)\right)+\cdots+(-1)^{\ell-1} i p^{t-\ell}\left(\sum_{a=\ell-1}^{t-1}\left(\begin{array}{c}
a \\
\ell-1
\end{array}\right)\right)+\cdots-i-\sum_{\ell=0}^{t-1} p^{\ell} \\
& =i p^{t-1}\left(\begin{array}{l}
t \\
1
\end{array}\right)+\cdots+(-1)^{\ell-1} i p^{t-\ell}\left(\begin{array}{l}
t \\
\ell
\end{array}\right)+\cdots-i-\sum_{\ell=0}^{t-1} p^{\ell}, \\
& =i \sum_{\ell=1}^{t}\left(\begin{array}{l}
t \\
\ell
\end{array}\right) p^{t-\ell}(-1)^{\ell-1}-\sum_{\ell=0}^{t-1} p^{\ell} .
\end{aligned}
$$

The fact

$$
\sum_{a=\ell-1}^{t-1}\left(\begin{array}{c}
a \\
\ell-1
\end{array}\right)=\left(\begin{array}{l}
t \\
\ell
\end{array}\right)
$$

can be easily proven using induction on $t$ and using the fact that

$$
\left(\begin{array}{l}
t \\
\ell
\end{array}\right)+\left(\begin{array}{c}
t \\
\ell-1
\end{array}\right)=\left(\begin{array}{c}
t+1 \\
\ell
\end{array}\right)
$$

Now, we have

$$
\begin{aligned}
\left|T_{t}\right| & =n-\sum_{j=0}^{t-1}\left|S_{j}\right| \\
& =i p^{t}-\sum_{\ell=0}^{t-1} p^{\ell}-\left(i \sum_{\ell=1}^{t}\left(\begin{array}{l}
t \\
\ell
\end{array}\right) p^{t-\ell}(-1)^{\ell-1}-\sum_{\ell=0}^{t-1} p^{\ell}\right), \\
& =i p^{t}+i \sum_{\ell=1}^{t}\left(\begin{array}{l}
t \\
\ell
\end{array}\right) p^{t-\ell}(-1)^{\ell}=i \cdot(p-1)^{t} .
\end{aligned}
$$

Lemma 8. Given a prime $p>3, n=i p^{t}-\sum_{\ell=0}^{t-1} p^{\ell}$ with $1 \leqslant i \leqslant p-1$, and the set $T=\{1,2, \ldots, n\}$; the set $T_{1}$ contains no $p$-AP with

$$
d \in\left\{1 \leqslant d_{1} \leqslant\lfloor(n-1) /(p-1)\rfloor: d_{1} \not \equiv 0 \quad(\bmod p)\right\} .
$$

Proof. Assume $T_{1}$ contains a $p$-AP $a, a+d, \ldots, a+(p-1) d$. Here $a \not \equiv 0(\bmod p)$. Suppose $a \equiv j(\bmod p)$ for some $1 \leqslant j \leqslant p-1$. Then $a+d(p-z) \equiv 0(\bmod p)$ for some $1 \leqslant z \leqslant p-1$ when $d z \equiv j(\bmod p)$.

For each $d \in\left\{1 \leqslant d_{1} \leqslant\lfloor(n-1) /(p-1)\rfloor: d_{1} \not \equiv 0(\bmod p)\right\}$,

$$
\bigcup_{z=1}^{p-1}\{d z \quad(\bmod p)\}=\{1,2, \ldots, p-1\}
$$

and so there exists $1 \leqslant z \leqslant p-1$ for any $1 \leqslant j \leqslant p-1$ such that $d z \equiv j(\bmod p)$. But, this is a contradiction as there is no number in $T_{1}$ which is divisible by $p$. Hence $T_{1}$ contains no $p$-AP with $d \in\left\{1 \leqslant d_{1} \leqslant\lfloor(n-1) /(p-1)\rfloor: d_{1} \not \equiv 0(\bmod p)\right\}$. 
Lemma 9. The set $T_{t}$ is $p$-AP free.

Proof. By construction, $T_{t}$ contains no $p$-AP with $d \in\left\{1, p, p^{2}, \ldots, p^{t}\right\}$. By Lemma $8, T_{t}$ does not contain a $p$-AP with any other $d$. Hence $T_{t}$ is $p$-AP free.

\subsection{A construction algorithm for $r(k, n)$}

In this section, we propose a greedy algorithm for construction of $k$-AP free subsequence of $1,2, \ldots, n$. We call this algorithm Bi-symmetric Greedy Algorithm (BGA) as it builds a fully symmetric subsequence that is $k$-AP free.

1. Take $T=\{1, n\}$.

2. Choose the smallest $j \in\{1,2, \ldots, n\}-T$ such that $T \cup\{j, n-j+1\}$ is $k$-AP free. Set $T=T \cup\{j, n-j+1\}$.

3. Repeat step 2 until no such $j$ can be found.

4. Output $T$.

Clearly,

$$
r(k, n) \geqslant|B G A(k, n)| .
$$

From experimental data, we have the following observation:

Observation 3. Consider a prime $p>3$. Then $|B G A(p, x)|=x$ if $1 \leqslant x \leqslant p-2$, or else for $1 \leqslant i \leqslant p-1$ and $t \geqslant 1$,

$$
\left|B G A\left(p, \frac{(i p-i-1) p^{t}+1}{(p-1)}\right)\right|=i \cdot(p-1)^{t} .
$$

See Appendix B for supporting data.

\section{Acknowledgements}

The authors would like to thank the anonymous referee and the Editor for helpful comments and suggestions.

\section{References}

[1] F. A. Behrend, On Sets of Integers Which Contain No Three Terms in Arithmetic Progression, Proc. Nat. Acad. Sci. USA, 32 (1946), 331-332.

[2] J. Dybizbański, Sequences containing no 3-term arithmetic progressions, Elec. J. of Comb., 19(2) (2012), \#P15.

[3] M. Elkin, An Improved Construction of Progression-Free Sets, Israeli J. Math., 184, (2011), 93-128. 
[4] P. Erdős and P. Turán, On some sequence of integers, J. London Math. Soc., 11 (1936), 261-264.

[5] K. F. Roth, On certain set of integers, J. London Math. Soc., 28 (1953), 104-109.

[6] R. Salem and D. C. Spencer, On Sets of Integers Which Contain No Three Terms in Arithmetic Progression, Proc. Nat. Acad. Sci. USA, 28 (1942), 561-563.

[7] A. Sharma, Sequences of Integers Avoiding 3-term Arithmetic Progressions, Elec. J. of Comb., 19 (2012), \#P27.

[8] E. Szemeredi, On sets of integers containing no $k$ elements in arithmetic progression, Acta Arithmetica, 27 (1975), 199-245. 


\section{A Computed values}

\begin{tabular}{|c|c|c|c|c|c|c|c|c|c|}
\hline$n$ & $c_{\max }(3, n)$ & $|U|$ & $n$ & $c_{\max }(3, n)$ & $|U|$ & $n$ & $c_{\max }(3, n)$ & $|U|$ & \\
\hline 4 & 2 & 2 & 8 & 6 & 2 & 12 & 10 & 2 & $\cdots$ \\
\hline 5 & 4 & 4 & 9 & 8 & 4 & 13 & 12 & 4 & $\cdots$ \\
\hline 6 & 4 & 2 & 10 & 8 & 2 & 14 & 12 & 2 & $\cdots$ \\
\hline 7 & 5 & 4 & 11 & 9 & 4 & 15 & 13 & 4 & $\cdots$ \\
\hline
\end{tabular}

$f(5)=8, c_{\max }(5, n-24)=c_{\max }(5, n)+20$ for $n \geqslant 62$

\begin{tabular}{c|c|c||c|c|c||c|c|c||c}
\hline$n$ & $c_{\max }(5, n)$ & $|U|$ & $n$ & $c_{\max }(5, n)$ & $|U|$ & $n$ & $c_{\max }(5, n)$ & $|U|$ & \\
\hline 38 & 29 & 6 & 62 & 49 & 6 & 86 & 69 & 6 & $\cdots$ \\
39 & 30 & 6 & 63 & 50 & 6 & 87 & 70 & 6 & $\cdots$ \\
40 & 31 & 6 & 64 & 51 & 6 & 88 & 71 & 6 & $\cdots$ \\
41 & 32 & 6 & 65 & 52 & 6 & 89 & 72 & 6 & $\cdots$ \\
42 & 33 & $\mathbf{8}$ & 66 & 53 & $\mathbf{8}$ & 90 & 73 & $\mathbf{8}$ & $\cdots$ \\
$\vdots$ & $\vdots$ & $\vdots$ & $\vdots$ & $\vdots$ & $\vdots$ & $\vdots$ & $\vdots$ & $\vdots$ & $\vdots$ \\
60 & 47 & 4 & 84 & 67 & 4 & 108 & 87 & 4 & $\cdots$ \\
61 & 49 & $\mathbf{8}$ & 85 & 69 & $\mathbf{8}$ & 109 & 89 & $\mathbf{8}$ & $\cdots$ \\
\hline
\end{tabular}

$f(7)=14, c_{\max }(7, n-120)=c_{\max }(7, n)+94$ for $n \geqslant 467$

\begin{tabular}{c|c|c||c|c|c||c|c|c||c}
\hline$n$ & $c_{\max }(7, n)$ & $|U|$ & $n$ & $c_{\max }(7, n)$ & $|U|$ & $n$ & $c_{\max }(7, n)$ & $|U|$ & \\
\hline 347 & 268 & 10 & 467 & 362 & 10 & 587 & 456 & 10 & $\ldots$ \\
348 & 269 & 10 & 468 & 363 & 10 & 588 & 457 & 10 & $\ldots$ \\
349 & 270 & 10 & 469 & 364 & 10 & 589 & 458 & 10 & $\ldots$ \\
350 & 271 & 10 & 470 & 365 & 10 & 590 & 459 & 10 & $\ldots$ \\
$\vdots$ & $\vdots$ & $\vdots$ & $\vdots$ & $\vdots$ & $\vdots$ & $\vdots$ & $\vdots$ & $\vdots$ & $\vdots$ \\
358 & 278 & $\mathbf{1 4}$ & 478 & 372 & $\mathbf{1 4}$ & 598 & 466 & $\mathbf{1 4}$ & $\ldots$ \\
$\vdots$ & $\vdots$ & $\vdots$ & $\vdots$ & $\vdots$ & $\vdots$ & $\vdots$ & $\vdots$ & $\vdots$ & $\vdots$ \\
465 & 361 & 10 & 585 & 455 & 10 & 705 & 549 & 10 & $\ldots$ \\
466 & 361 & $\mathbf{1 4}$ & 586 & 455 & $\mathbf{1 4}$ & 706 & 549 & $\mathbf{1 4}$ & $\ldots$ \\
\hline
\end{tabular}

$f(9)=20, c_{\max }(9, n-6720)=c_{\max }(9, n)+5104$ for $n \geqslant 13365$

\begin{tabular}{|c|c|c|c|c|c|c|c|c|c|}
\hline$n$ & $c_{\max }(9, n)$ & $|U|$ & $n$ & $c_{\max }(9, n)$ & $|U|$ & $n$ & $c_{\max }(9, n)$ & $|U|$ & \\
\hline 6645 & 5043 & 14 & 13365 & 10147 & 14 & 20085 & 15251 & 14 & $\cdots$ \\
\hline 6646 & 5045 & 14 & 13366 & 10149 & 14 & 20086 & 15253 & 14 & $\cdots$ \\
\hline 6647 & 5045 & 16 & 13367 & 10149 & 16 & 20087 & 15253 & 16 & $\cdots$ \\
\hline 6648 & 5045 & 14 & 13368 & 10149 & 14 & 20088 & 15253 & 14 & $\cdots$ \\
\hline$\vdots$ & : & $\vdots$ & $\vdots$ & : & t & : & : & : & \\
\hline 6691 & 5078 & 20 & 13411 & 10182 & 20 & 20131 & 15286 & 20 & $\cdots$ \\
\hline$\vdots$ & $\vdots$ & $\vdots$ & $\vdots$ & : & : & : & $\vdots$ & ( & : \\
\hline 13363 & 10146 & 14 & 20083 & 15250 & 14 & 26803 & 20354 & 14 & $\cdots$ \\
\hline 13364 & 10146 & 16 & 20084 & 15250 & 16 & 26804 & 20354 & 16 & $\cdots$ \\
\hline
\end{tabular}

$f(11)=26, f(13)=36$ 


\section{B BGA results}

$$
p=3
$$

$t=1, i=1,|B G A(3,2)|=2$

$B G A(3,2) \mid=\{1,2\}$

$t=2, i=1,|B G A(3,5)|=4$

$B G A(3,5) \mid=\{1,2,4,5\}$

$t=3, i=1,|B G A(3,14)|=8$

$B G A(3,14) \mid=\{1,2,4,5,10,11,13,14\}$

$t=4, i=1,|B G A(3,41)|=16$

$B G A(3,41) \mid=\{1,2,4,5,10,11,13,14,28,29,31,32,37,38,40,41\}$

$t=5, i=1,|B G A(3,122)|=32$

$B G A(3,122) \mid=\{1,2,4,5,10,11,13,14,28,29,31,32,37,38,40,41,82,83,85,86,91,92,94,95,109,110$, $112,113,118,119,121,122\}$

$t=6, i=1,|B G A(3,365)|=64$

$B G A(3,365) \mid=\{1,2,4,5,10,11,13,14,28,29,31,32,37,38,40,41,82,83,85,86,91,92,94,95,109,110$, $112,113,118,119,121,122,244,245,247,248,253,254,256,257,271,272,274,275,280,281,283,284,325$, $326,328,329,334,335,337,338,352,353,355,356,361,362,364,365\}$

$t=7, i=1,|B G A(3,1094)|=128$

$B G A(3,1094) \mid=\{1,2,4,5,10,11,13,14,28,29,31,32,37,38,40,41,82,83,85,86,91,92,94,95,109,110$, $112,113,118,119,121,122,244,245,247,248,253,254,256,257,271,272,274,275,280,281,283,284,325$, $326,328,329,334,335,337,338,352,353,355,356,361,362,364,365,730,731,733,734,739,740,742,743$, $757,758,760,761,766,767,769,770,811,812,814,815,820,821,823,824,838,839,841,842,847,848,850$, $851,973,974,976,977,982,983,985,986,1000,1001,1003,1004,1009,1010,1012,1013,1054,1055,1057$, $1058,1063,1064,1066,1067,1081,1082,1084,1085,1090,1091,1093,1094\}$

$$
p=5
$$

$t=1, i=1,|B G A(5,4)|=4$

$B G A(5,4) \mid=\{1,2,3,4\}$

$t=1, i=2,|B G A(5,9)|=8$

$B G A(5,9) \mid=\{1,2,3,4,6,7,8,9\}$

$t=1, i=3,|B G A(5,14)|=12$

$B G A(5,14) \mid=\{1,2,3,4,6,7,8,9,11,12,13,14\}$

$t=2, i=1,|B G A(5,19)|=16$

$B G A(5,19) \mid=\{1,2,3,4,6,7,8,9,11,12,13,14,16,17,18,19\}$

$t=2, i=2,|B G A(5,44)|=32$

$B G A(5,44) \mid=\{1,2,3,4,6,7,8,9,11,12,13,14,16,17,18,19,26,27,28,29,31,32,33,34,36,37,38,39,41$, $42,43,44\}$

$t=2, i=3,|B G A(5,69)|=48$

$B G A(5,69) \mid=\{1,2,3,4,6,7,8,9,11,12,13,14,16,17,18,19,26,27,28,29,31,32,33,34,36,37,38,39,41$, $42,43,44,51,52,53,54,56,57,58,59,61,62,63,64,66,67,68,69\}$

$t=3, i=1,|B G A(5,94)|=64$ 
$B G A(5,94) \mid=\{1,2,3,4,6,7,8,9,11,12,13,14,16,17,18,19,26,27,28,29,31,32,33,34,36,37,38,39,41$, $42,43,44,51,52,53,54,56,57,58,59,61,62,63,64,66,67,68,69,76,77,78,79,81,82,83,84,86,87,88,89$,

$91,92,93,94\}$

$t=3, i=2,|B G A(5,219)|=128$

$B G A(5,219) \mid=\{1,2,3,4,6,7,8,9,11,12,13,14,16,17,18,19,26,27,28,29,31,32,33,34,36,37,38,39,41$, $42,43,44,51,52,53,54,56,57,58,59,61,62,63,64,66,67,68,69,76,77,78,79,81,82,83,84,86,87,88,89$, $91,92,93,94,126,127,128,129,131,132,133,134,136,137,138,139,141,142,143,144,151,152,153,154$, $156,157,158,159,161,162,163,164,166,167,168,169,176,177,178,179,181,182,183,184,186,187,188$, $189,191,192,193,194,201,202,203,204,206,207,208,209,211,212,213,214,216,217,218,219\}$

$$
p=7
$$

$t=1, i=1,|B G A(7,6)|=6$

$B G A(7,6) \mid=\{1,2,3,4,5,6\}$

$t=1, i=2,|B G A(7,13)|=12$

$B G A(7,13) \mid=\{1,2,3,4,5,6,8,9,10,11,12,13\}$

$t=1, i=3,|B G A(7,20)|=18$

$B G A(7,20) \mid=\{1,2,3,4,5,6,8,9,10,11,12,13,15,16,17,18,19,20\}$

$t=1, i=4,|B G A(7,27)|=24$

$B G A(7,27) \mid=\{1,2,3,4,5,6,8,9,10,11,12,13,15,16,17,18,19,20,22,23,24,25,26,27\}$

$t=1, i=5,|B G A(7,34)|=30$

$B G A(7,34) \mid=\{1,2,3,4,5,6,8,9,10,11,12,13,15,16,17,18,19,20,22,23,24,25,26,27,29,30,31,32,33$,

$34\}$

$t=2, i=1,|B G A(7,41)|=36$

$B G A(7,41) \mid=\{1,2,3,4,5,6,8,9,10,11,12,13,15,16,17,18,19,20,22,23,24,25,26,27,29,30,31,32,33$, $34,36,37,38,39,40,41\}$

$t=2, i=2,|B G A(7,90)|=72$

$B G A(7,90) \mid=\{1,2,3,4,5,6,8,9,10,11,12,13,15,16,17,18,19,20,22,23,24,25,26,27,29,30,31,32,33$, $34,36,37,38,39,40,41,50,51,52,53,54,55,57,58,59,60,61,62,64,65,66,67,68,69,71,72,73,74,75,76$,

$78,79,80,81,82,83,85,86,87,88,89,90\}$

$t=2, i=3,|B G A(7,139)|=108$

$B G A(7,139) \mid=\{1,2,3,4,5,6,8,9,10,11,12,13,15,16,17,18,19,20,22,23,24,25,26,27,29,30,31,32,33$, $34,36,37,38,39,40,41,50,51,52,53,54,55,57,58,59,60,61,62,64,65,66,67,68,69,71,72,73,74,75,76$, $78,79,80,81,82,83,85,86,87,88,89,90,99,100,101,102,103,104,106,107,108,109,110,111,113,114$, $115,116,117,118,120,121,122,123,124,125,127,128,129,130,131,132,134,135,136,137,138,139\}$

$$
p=11
$$

$t=1, i=1,|B G A(11,10)|=10$

$B G A(11,10) \mid=\{1,2,3,4,5,6,7,8,9,10\}$

$t=1, i=2,|B G A(11,21)|=20$

$B G A(11,21) \mid=\{1,2,3,4,5,6,7,8,9,10,12,13,14,15,16,17,18,19,20,21\}$ 
$t=1, i=3,|B G A(11,32)|=30$

$B G A(11,32) \mid=\{1,2,3,4,5,6,7,8,9,10,12,13,14,15,16,17,18,19,20,21,23,24,25,26,27,28,29,30,31$, $32\}$

$t=1, i=4,|B G A(11,43)|=40$

$B G A(11,43) \mid=\{1,2,3,4,5,6,7,8,9,10,12,13,14,15,16,17,18,19,20,21,23,24,25,26,27,28,29,30,31$, $32,34,35,36,37,38,39,40,41,42,43\}$

$t=1, i=5,|B G A(11,54)|=50$

$B G A(11,54) \mid=\{1,2,3,4,5,6,7,8,9,10,12,13,14,15,16,17,18,19,20,21,23,24,25,26,27,28,29,30,31$, $32,34,35,36,37,38,39,40,41,42,43,45,46,47,48,49,50,51,52,53,54\}$

$t=1, i=6,|B G A(11,65)|=60$

$B G A(11,65) \mid=\{1,2,3,4,5,6,7,8,9,10,12,13,14,15,16,17,18,19,20,21,23,24,25,26,27,28,29,30,31$, $32,34,35,36,37,38,39,40,41,42,43,45,46,47,48,49,50,51,52,53,54,56,57,58,59,60,61,62,63,64,65\}$ $t=1, i=7,|B G A(11,76)|=70$

$B G A(11,76) \mid=\{1,2,3,4,5,6,7,8,9,10,12,13,14,15,16,17,18,19,20,21,23,24,25,26,27,28,29,30,31$, $32,34,35,36,37,38,39,40,41,42,43,45,46,47,48,49,50,51,52,53,54,56,57,58,59,60,61,62,63,64,65$, $67,68,69,70,71,72,73,74,75,76\}$

$t=1, i=8,|B G A(11,87)|=80$

$B G A(11,87) \mid=\{1,2,3,4,5,6,7,8,9,10,12,13,14,15,16,17,18,19,20,21,23,24,25,26,27,28,29,30,31$, $32,34,35,36,37,38,39,40,41,42,43,45,46,47,48,49,50,51,52,53,54,56,57,58,59,60,61,62,63,64,65$, $67,68,69,70,71,72,73,74,75,76,78,79,80,81,82,83,84,85,86,87\}$

$t=1, i=9,|B G A(11,98)|=90$

$B G A(11,98) \mid=\{1,2,3,4,5,6,7,8,9,10,12,13,14,15,16,17,18,19,20,21,23,24,25,26,27,28,29,30,31$, $32,34,35,36,37,38,39,40,41,42,43,45,46,47,48,49,50,51,52,53,54,56,57,58,59,60,61,62,63,64,65$, $67,68,69,70,71,72,73,74,75,76,78,79,80,81,82,83,84,85,86,87,89,90,91,92,93,94,95,96,97,98\}$ $t=2, i=1,|B G A(11,109)|=100$ $B G A(11,109) \mid=\{1,2,3,4,5,6,7,8,9,10,12,13,14,15,16,17,18,19,20,21,23,24,25,26,27,28,29,30,31$, $32,34,35,36,37,38,39,40,41,42,43,45,46,47,48,49,50,51,52,53,54,56,57,58,59,60,61,62,63,64,65$, $67,68,69,70,71,72,73,74,75,76,78,79,80,81,82,83,84,85,86,87,89,90,91,92,93,94,95,96,97,98,100$, $101,102,103,104,105,106,107,108,109\}$

$t=2, i=2,|B G A(11,230)|=200$

$B G A(11,230) \mid=\{1,2,3,4,5,6,7,8,9,10,12,13,14,15,16,17,18,19,20,21,23,24,25,26,27,28,29,30,31$, $32,34,35,36,37,38,39,40,41,42,43,45,46,47,48,49,50,51,52,53,54,56,57,58,59,60,61,62,63,64,65$, $67,68,69,70,71,72,73,74,75,76,78,79,80,81,82,83,84,85,86,87,89,90,91,92,93,94,95,96,97,98,100$, 101, 102, 103, 104, 105, 106, 107, 108, 109, 122, 123, 124, 125, 126, 127, 128, 129, 130, 131, 133, 134, 135, 136, 137, 138, 139, 140, 141, 142, 144, 145, 146, 147, 148, 149, 150, 151, 152, 153, 155, 156, 157, 158, 159, 160, 161, $162,163,164,166,167,168,169,170,171,172,173,174,175,177,178,179,180,181,182,183,184,185,186$, $188,189,190,191,192,193,194,195,196,197,199,200,201,202,203,204,205,206,207,208,210,211,212$, $213,214,215,216,217,218,219,221,222,223,224,225,226,227,228,229,230\}$ 


\section{Computed values of $r(k, n)$}

\begin{tabular}{c|c|c|c|c||c|c|c|c|c}
\hline$n$ & $b(n)$ & $\begin{array}{c}\text { lower bound } \\
|B G A(3, n)|\end{array}$ & $r(3, n)$ & $\begin{array}{c}\text { upper bound } \\
\text { (Theorem } 1)\end{array}$ & $n$ & $b(n)$ & $\begin{array}{c}\text { lower bound } \\
|B G A(3, n)|\end{array}$ & $r(3, n)$ & $\begin{array}{c}\text { upper bound } \\
\text { (Theorem } 1)\end{array}$ \\
\hline 4 & 2 & 2 & 3 & 3 & 54 & 2 & 16 & 18 & 41 \\
5 & 1 & 4 & 4 & 4 & 58 & 2 & 16 & 19 & 44 \\
9 & 4 & 4 & 5 & 7 & 63 & 2 & 16 & 20 & 48 \\
11 & 7 & 6 & 6 & 9 & 71 & 4 & 18 & 21 & 54 \\
13 & 6 & 6 & 7 & 10 & 74 & 1 & 18 & 22 & 56 \\
14 & 1 & 8 & 8 & 11 & 82 & 10 & 18 & 23 & 62 \\
20 & 2 & 8 & 9 & 15 & 84 & 1 & 18 & 24 & 63 \\
24 & 2 & 8 & 10 & 18 & 92 & 14 & 22 & 25 & 69 \\
26 & 2 & 10 & 11 & 20 & 95 & 8 & 24 & 26 & 72 \\
30 & 1 & 10 & 12 & 23 & 100 & 2 & 24 & 27 & 75 \\
32 & 2 & 12 & 13 & 24 & 104 & 1 & 24 & 28 & 78 \\
36 & 2 & 12 & 14 & 27 & 111 & 6 & 26 & 29 & 84 \\
40 & 20 & 14 & 15 & 30 & 114 & 1 & 28 & 30 & 86 \\
41 & 1 & 16 & 16 & 31 & 121 & 70 & 30 & 31 & 91 \\
51 & 14 & 16 & 17 & 39 & 122 & 1 & 32 & 32 & 92 \\
\hline
\end{tabular}

\begin{tabular}{c|c|c|c|c||c|c|c|c|c}
\hline$n$ & $b(n)$ & $\begin{array}{c}\text { lower bound } \\
|B G A(4, n)|\end{array}$ & $r(4, n)$ & $\begin{array}{c}\text { upper bound } \\
\text { (Theorem } 1 \text { ) }\end{array}$ & $n$ & $b(n)$ & $\begin{array}{c}\text { lower bound } \\
|B G A(4, n)|\end{array}$ & $r(4, n)$ & $\begin{array}{c}\text { upper bound } \\
\text { (Theorem 1 }\end{array}$ \\
\hline 5 & 3 & 4 & 4 & 5 & 30 & 2 & 16 & 18 & 25 \\
6 & 2 & 4 & 5 & 5 & 33 & 6 & 16 & 19 & 28 \\
8 & 4 & 6 & 6 & 7 & 34 & 2 & 18 & 20 & 29 \\
9 & 2 & 6 & 7 & 8 & 37 & 6 & 18 & 21 & 31 \\
10 & 1 & 8 & 8 & 9 & 40 & 14 & 18 & 22 & 34 \\
13 & 3 & 9 & 9 & 11 & 43 & 38 & 18 & 23 & 36 \\
15 & 2 & 8 & 10 & 13 & 45 & 2 & 20 & 24 & 38 \\
17 & 10 & 10 & 11 & 15 & 48 & 2 & 20 & 25 & 40 \\
19 & 24 & 12 & 12 & 16 & 50 & 3 & 22 & 26 & 42 \\
21 & 10 & 12 & 13 & 18 & 53 & 12 & 22 & 27 & 45 \\
23 & 12 & 13 & 14 & 20 & 54 & 1 & 24 & 28 & 45 \\
25 & 2 & 14 & 15 & 21 & 58 & 6 & 26 & 29 & 49 \\
27 & 8 & 14 & 16 & 23 & 60 & 1 & 26 & 30 & 50 \\
28 & 4 & 14 & 17 & 24 & & & & & \\
\hline
\end{tabular}




\begin{tabular}{c|c|c|c|c||c|c|c|c|c}
\hline$n$ & $b(n)$ & $\begin{array}{c}\text { lower bound } \\
|B G A(5, n)|\end{array}$ & $r(5, n)$ & $\begin{array}{c}\text { upper bound } \\
\text { (Theorem } 1)\end{array}$ & $n$ & $b(n)$ & $\begin{array}{c}\text { lower bound } \\
|B G A(5, n)|\end{array}$ & $r(5, n)$ & $\begin{array}{c}\text { upper bound } \\
\text { (Theorem } 1)\end{array}$ \\
\hline 6 & 4 & 4 & 5 & 6 & 52 & 2 & 32 & 35 & 46 \\
7 & 3 & 6 & 6 & 7 & 54 & 1300 & 32 & 36 & 48 \\
8 & 2 & 6 & 7 & 7 & 56 & 3508 & 32 & 37 & 49 \\
9 & 1 & 8 & 8 & 8 & 57 & 1736 & 36 & 38 & 50 \\
11 & 6 & 8 & 9 & 10 & 58 & 768 & 32 & 39 & 51 \\
12 & 4 & 10 & 10 & 11 & 59 & 256 & 40 & 40 & 52 \\
13 & 2 & 8 & 11 & 12 & 61 & 512 & 36 & 41 & 54 \\
14 & 1 & 12 & 12 & 13 & 62 & 192 & 38 & 42 & 55 \\
16 & 4 & 12 & 13 & 14 & 63 & 64 & 38 & 43 & 56 \\
17 & 3 & 12 & 14 & 15 & 64 & 16 & 36 & 44 & 56 \\
18 & 2 & 14 & 15 & 16 & 66 & 32 & 40 & 45 & 58 \\
19 & 1 & 16 & 16 & 17 & 67 & 12 & 40 & 46 & 59 \\
24 & 40 & 16 & 17 & 21 & 68 & 4 & 42 & 47 & 60 \\
25 & 2 & 16 & 18 & 22 & 69 & 1 & 48 & 48 & 61 \\
27 & 70 & 18 & 19 & 24 & 76 & 2012 & 42 & 49 & 67 \\
28 & 12 & 18 & 20 & 25 & 77 & 1202 & 44 & 50 & 68 \\
29 & 2 & 20 & 21 & 26 & 78 & 640 & 44 & 51 & 69 \\
31 & 5 & 20 & 22 & 28 & 79 & 256 & 48 & 52 & 70 \\
33 & 266 & 22 & 23 & 29 & 81 & 768 & 44 & 53 & 71 \\
34 & 81 & 24 & 24 & 30 & 82 & 432 & 48 & 54 & 72 \\
36 & 236 & 24 & 25 & 32 & 83 & 216 & 48 & 55 & 73 \\
37 & 115 & 26 & 26 & 33 & 84 & 81 & 52 & 56 & 74 \\
38 & 48 & 24 & 27 & 34 & 86 & 216 & 50 & 57 & 76 \\
39 & 16 & 28 & 28 & 35 & 87 & 108 & 52 & 58 & 77 \\
41 & 32 & 26 & 29 & 36 & 88 & 48 & 50 & 59 & 77 \\
42 & 12 & 28 & 30 & 37 & 89 & 16 & 56 & 60 & 78 \\
43 & 4 & 30 & 31 & 38 & 91 & 32 & 54 & 61 & 80 \\
44 & 1 & 32 & 32 & 39 & 92 & 12 & 56 & 62 & 81 \\
49 & 2 & 32 & 33 & 43 & 93 & 4 & 56 & 63 & 82 \\
51 & 18 & 32 & 34 & 45 & 94 & 1 & 64 & 64 & 83 \\
\hline
\end{tabular}

THE ELECTRONIC JOURNAL OF COMBINATORICS 20(4) (2013), \#P29 


\begin{tabular}{c|c|c|c|c||c|c|c|c|c}
\hline$n$ & $b(n)$ & $\begin{array}{c}\text { lower bound } \\
|B G A(7, n)|\end{array}$ & $r(7, n)$ & $\begin{array}{c}\text { upper bound } \\
\text { (Theorem } 1)\end{array}$ & $n$ & $b(n)$ & $\begin{array}{c}\text { lower bound } \\
|B G A(7, n)|\end{array}$ & $r(7, n)$ & $\begin{array}{c}\text { upper bound } \\
\text { (Theorem } 1)\end{array}$ \\
\hline 8 & 6 & 6 & 7 & 8 & 51 & 76 & 38 & 40 & 47 \\
9 & 5 & 8 & 8 & 9 & 52 & 22 & 38 & 41 & 48 \\
10 & 4 & 8 & 9 & 10 & 53 & 2 & 40 & 42 & 49 \\
11 & 3 & 10 & 10 & 11 & 55 & 242 & 42 & 43 & 51 \\
12 & 2 & 10 & 11 & 11 & 56 & 8 & 42 & 44 & 52 \\
13 & 1 & 12 & 12 & 12 & 57 & 4 & 42 & 45 & 53 \\
15 & 12 & 12 & 13 & 14 & 59 & 218 & 44 & 46 & 55 \\
16 & 9 & 14 & 14 & 15 & 60 & 54 & 46 & 47 & 55 \\
17 & 6 & 13 & 15 & 16 & 61 & 10 & 46 & 48 & 56 \\
18 & 4 & 16 & 16 & 17 & 63 & 32 & 48 & 49 & 58 \\
19 & 2 & 16 & 17 & 18 & 64 & 14 & 48 & 50 & 59 \\
20 & 1 & 18 & 18 & 19 & 65 & 2 & 50 & 51 & 60 \\
22 & 8 & 18 & 19 & 21 & 67 & 19807 & 52 & 52 & 62 \\
23 & 6 & 20 & 20 & 22 & 68 & 10294 & 52 & 53 & 63 \\
24 & 4 & 20 & 21 & 22 & 69 & 4103 & 54 & 54 & 64 \\
25 & 3 & 20 & 22 & 23 & 71 & 18522 & 54 & 55 & 66 \\
26 & 2 & 20 & 23 & 24 & 72 & 11541 & 56 & 56 & 66 \\
27 & 1 & 24 & 24 & 25 & 73 & 6914 & 54 & 57 & 67 \\
29 & 6 & 22 & 25 & 27 & 74 & 3888 & 56 & 58 & 68 \\
30 & 5 & 24 & 26 & 28 & 75 & 1944 & 56 & 59 & 69 \\
31 & 4 & 24 & 27 & 29 & 76 & 729 & 60 & 60 & 70 \\
32 & 3 & 24 & 28 & 30 & 78 & 2918 & 58 & 61 & 72 \\
33 & 2 & 26 & 29 & 31 & 79 & 1621 & 62 & 62 & 73 \\
34 & 1 & 30 & 30 & 32 & 80 & 864 & 60 & 63 & 74 \\
36 & 6 & 30 & 31 & 33 & 81 & 432 & 60 & 64 & 75 \\
37 & 5 & 28 & 32 & 34 & 82 & 192 & 62 & 65 & 76 \\
38 & 4 & 30 & 33 & 35 & 83 & 64 & 66 & 66 & 77 \\
39 & 3 & 32 & 34 & 36 & 85 & 192 & 64 & 67 & 78 \\
40 & 2 & 30 & 35 & 37 & 86 & 80 & 64 & 68 & 79 \\
41 & 1 & 36 & 36 & 38 & 87 & 32 & 66 & 69 & 80 \\
46 & 18 & 36 & 37 & 43 & 88 & 12 & 68 & 70 & 81 \\
48 & 9 & 36 & 38 & 44 & 89 & 4 & 66 & 71 & 82 \\
50 & 392 & 36 & 39 & 46 & 90 & 1 & 72 & 72 & 83 \\
\hline & & & & & & & & & \\
\end{tabular}

THe ELeCtronic Journal of COMBinatorics 20(4) (2013), \#P29 


\begin{tabular}{|c|c|c|c|c|c|c|c|c|c|}
\hline$n$ & $b(n)$ & $\begin{array}{l}\text { lower bound } \\
|B G A(11, n)|\end{array}$ & $r(11, n)$ & $\begin{array}{l}\text { upper bound } \\
\text { (Theorem 1) }\end{array}$ & $n$ & $b(n)$ & $\begin{array}{l}\text { lower bound } \\
|B G A(11, n)|\end{array}$ & $r(11, n)$ & $\begin{array}{l}\text { upper bound } \\
\text { (Theorem 1) }\end{array}$ \\
\hline 12 & 10 & 10 & 11 & 12 & 61 & 5 & 54 & 56 & 58 \\
\hline 13 & 9 & 12 & 12 & 13 & 62 & 4 & 52 & 57 & 59 \\
\hline 14 & 8 & 12 & 13 & 14 & 63 & 3 & 54 & 58 & 60 \\
\hline 15 & 7 & 14 & 14 & 15 & 64 & 2 & 56 & 59 & 61 \\
\hline 16 & 6 & 14 & 15 & 16 & 65 & 1 & 60 & 60 & 62 \\
\hline 17 & 5 & 16 & 16 & 17 & 67 & 10 & 56 & 61 & 64 \\
\hline 18 & 4 & 16 & 17 & 18 & 68 & 9 & 58 & 62 & 65 \\
\hline 19 & 3 & 18 & 18 & 19 & 69 & 8 & 58 & 63 & 66 \\
\hline 20 & 2 & 18 & 19 & 19 & 70 & 7 & 62 & 64 & 67 \\
\hline 21 & 1 & 20 & 20 & 20 & 71 & 6 & 62 & 65 & 68 \\
\hline 23 & 30 & 20 & 21 & 22 & 72 & 5 & 60 & 66 & 69 \\
\hline 24 & 25 & 22 & 22 & 23 & 73 & 4 & 62 & 67 & 70 \\
\hline 25 & 20 & 21 & 23 & 24 & 74 & 3 & 62 & 68 & 71 \\
\hline 26 & 16 & 24 & 24 & 25 & 75 & 2 & 66 & 69 & 72 \\
\hline 27 & 12 & 24 & 25 & 26 & 76 & 1 & 70 & 70 & 73 \\
\hline 28 & 9 & 26 & 26 & 27 & 78 & 10 & 66 & 71 & 75 \\
\hline 29 & 6 & 25 & 27 & 28 & 79 & 9 & 66 & 72 & 76 \\
\hline 30 & 4 & 28 & 28 & 29 & 80 & 8 & 68 & 73 & 76 \\
\hline 31 & 2 & 28 & 29 & 30 & 81 & 7 & 68 & 74 & 77 \\
\hline 32 & 1 & 30 & 30 & 31 & 82 & 6 & 68 & 75 & 78 \\
\hline 34 & 20 & 30 & 31 & 33 & 83 & 5 & 72 & 76 & 79 \\
\hline 35 & 16 & 32 & 32 & 34 & 84 & 4 & 72 & 77 & 80 \\
\hline 36 & 12 & 32 & 33 & 35 & 85 & 3 & 74 & 78 & 81 \\
\hline 37 & 9 & 32 & 34 & 36 & 86 & 2 & 74 & 79 & 82 \\
\hline 38 & 6 & 34 & 35 & 37 & 87 & 1 & 80 & 80 & 83 \\
\hline 39 & 5 & 34 & 36 & 38 & 89 & 10 & 76 & 81 & 85 \\
\hline 40 & 4 & 36 & 37 & 38 & 90 & 9 & 78 & 82 & 86 \\
\hline 41 & 3 & 36 & 38 & 39 & 91 & 8 & 76 & 83 & 87 \\
\hline 42 & 2 & 38 & 39 & 40 & 92 & 7 & 80 & 84 & 88 \\
\hline 43 & 1 & 40 & 40 & 41 & 93 & 6 & 80 & 85 & 89 \\
\hline 45 & 10 & 39 & 41 & 43 & 94 & 5 & 80 & 86 & 90 \\
\hline 46 & 9 & 40 & 42 & 44 & 95 & 4 & 82 & 87 & 91 \\
\hline 47 & 8 & 40 & 43 & 45 & 96 & 3 & 80 & 88 & 92 \\
\hline 48 & 7 & 42 & 44 & 46 & 97 & 2 & 80 & 89 & 93 \\
\hline 49 & 6 & 42 & 45 & 47 & 98 & 1 & 90 & 90 & 94 \\
\hline 50 & 5 & 44 & 46 & 48 & 100 & 10 & 84 & 91 & 95 \\
\hline 51 & 4 & 43 & 47 & 49 & 101 & 9 & 84 & 92 & 96 \\
\hline 52 & 3 & 44 & 48 & 50 & 102 & 8 & 88 & 93 & 97 \\
\hline 53 & 2 & 46 & 49 & 51 & 103 & 7 & 86 & 94 & 98 \\
\hline 54 & 1 & 50 & 50 & 52 & 104 & 6 & 88 & 95 & 99 \\
\hline 56 & 10 & 48 & 51 & 54 & 105 & 5 & 90 & 96 & 100 \\
\hline 57 & 9 & 50 & 52 & 55 & 106 & 4 & 90 & 97 & 101 \\
\hline 58 & 8 & 50 & 53 & 56 & 107 & 3 & 92 & 98 & 102 \\
\hline 59 & 7 & 50 & 54 & 57 & 108 & 2 & 92 & 99 & 103 \\
\hline 60 & 6 & 52 & 55 & 57 & 109 & 1 & 100 & 100 & 104 \\
\hline
\end{tabular}

